\title{
miR-100-5p Enhanced Cell Cycle-mediated Chemoresistance Through Modulating CTDSPL/pRB/E2F1 Signaling Pathway in Oxaliplatin-Resistant Colorectal Cancer
}

\section{Ming-Cheng Chen}

Department of Surgery, Division of Colorectal Surgery, Taichung Veterans General Hospital, Taichung, Taiwan.

\section{Hema Sri Devi}

Cardiovascular and Mitochondrial Related Disease Research Center, Hualien Tzu Chi Hospital, Buddhist Tzu Chi Medical Foundation, Hualien, Taiwan

\section{Chaouhan Hitesh Singh}

Cardiovascular and Mitochondrial Related Disease Research Center, Hualien Tzu Chi Hospital, Buddhist Tzu Chi Medical Foundation, Hualien, Taiwan

\section{Tso-Fu Wang}

Department of Hematology and Oncology, Hualien Tzu Chi Hospital, Buddhist Tzu Chi Medical

Foundation, Hualien, Taiwan

\section{Chi-Cheng Li}

School of Medicine Tzu Chi University, 701, Section 3, Chung-Yang Road, Hualien 97004, Taiwan

\section{Tsung-Jung Ho}

Department of Chinese Medicine, Hualien Tzu Chi Hospital, Buddhist Tzu Chi Medical Foundation, Tzu Chi University, Hualien, Taiwan

\section{Marthandam Asokan Shibu}

Department of Biotechnology, Bharathiar University, Coimbatore, 641046, India

\section{Wei-Wen Kuo}

Department of Biological Science and Technology, China Medical University, Taichung, Taiwan

\section{Tzu-Ching Shih}

Department of Biomedical Imaging and Radiological Science, China Medical University, Taichung,

Taiwan.

\section{Yu-Jung Lin}

Cardiovascular and Mitochondrial Related Disease Research Center, Hualien Tzu Chi Hospital, Buddhist Tzu Chi Medical Foundation, Hualien, Taiwan

\section{Chih-Yang Huang ( $\boldsymbol{D}$ cyhuang@mail.cmu.edu.tw)}

Cardiovascular and Mitochondrial Related Disease Research Center, Hualien Tzu Chi Hospital, Buddhist Tzu Chi Medical Foundation, Hualien, Taiwan 


\section{Research Article}

Keywords: miR-100-5p, CTDSPL, p-RB, E2F1, FOXP3, chemoresistance, colorectal cancers

Posted Date: February 25th, 2022

DOl: https://doi.org/10.21203/rs.3.rs-1360100/v1

License: (c) (i) This work is licensed under a Creative Commons Attribution 4.0 International License. Read Full License 


\section{Abstract}

Purpose: miR-100-5p has been found upregulated in Oxaliplatin-resistant LoVo colorectal cell lines, but its function still need further study. Here, we investigated its function in cell regulation chemoresistance in chemoresistant colorectal cancer cell lines.

Methods: Microarray, quantitative polymerase chain reaction (qRT-PCR) and westernblot were used to analysis the level of miR-100-5p. The downstream candidate gen target of miR-100-5p, CTDSPL in Oxaliplatin resistant (OXR)-LoVo colorectal cell lines were screened using the different types of bioinformatics based miRNA target prediction data are TargetScan (http://www.targetscan.org/), miRanda (http://www.microrna.org/ and mirTar (http://mirtar.mbc.nctu.edu.tw/human/). CTDSPL, a phosphatase-like tumor suppressor level was detected by westernblot as well with cell cycle progression chemoresistance associated colorectal cancer cell CTDSPL/p-RB/E2F1. Immunofluorescence assay and westernblot showed the cell properties were altered between parental and Oxaliplatin resistant LoVo cell lines. Bioinformatic prediction, (http://alggen.1si.upc.es/cgi-bin/promo_v3) was used to extend the investigation of miR-100-5p upstream regulation through checking dissimilarity index with margin less or equal than 15\%, FOXP3, trancription factor forkhead box as upstream target. Interaction between miR100-5p and CTDSPL regulation was confirmed using mimic miR-100-5p overexpression and knockdown upstream target sh-RNA approach.

Results: hsa-miR-100-5p level was found significantly upregulated in LoVo-OXR than parental LoVo cell line, resulted in downregulation CTDSPL as a major target of miR-100-5p. The downregulated of CTDSPL/p-RB/E2F1 promotes cell proliferation and cell cycle progression in LoVo OXR compared with LoVo parental cell lines. Moreover, among various upstream targets FOXP3 is an upstream regulator of miR-100-5p. In addition, mimic miR-100-5p overexpression downregulated CTDSPL/p-RB/E2F1 signaling pathway was reverted upon miR-100-5p inhibition in CRC cell lines.

Conclusion: Collectively, miR-100-5p is chemoresistance inducer through modulation of CTDSPL/p$\mathrm{RB} / \mathrm{E} 2 \mathrm{~F} 1$ signaling pathway in CRC cell. This phenomenon might offer a therapeutic platform for overcoming chemoresistance in cancer treatment.

\section{Introduction}

According to global cancer statistics report 2018, colorectal cancer is in the rank 3rd of common cancer which found in the males and fourth most commonly diagnosed cancer in the females: it leads cancer related death in the worldwide, especially in the Western countries [1, 2]. Although a growing number of clinical advancement for the colorectal cancer treatment, the adjuvant systemic chemotherapy with cytotoxic drugs is recommended as optimal palliative therapeutic approaches for treatment of patients with severe CRC, recurrence and as the first line of therapeutic options for patients with metastatic relapse and spread [3]. While using the first-line and second-line chemotherapeutic treatment for $\mathrm{CRC}$, the combination of 5-fluorouracil (5-FU) with oxaliplatin (L-OHP) or irinotecan (CPT-11) $[4,5]$, is generally 
more effective and has significantly improved the 5 year survival rate for severe CRC cancer patient greater than $65 \%$. However, most of the CRC patients ultimately develop resistance conditions against chemotherapy agents. Hence, by understanding the molecular mechanisms associated with chemoresistance is a key strategies to attenuate the resistant condition who do not well respond to such type of treatment.

miRNAs are generally non-coding consist 19-22 nucleotide endogenous RNA molecules which is negatively regulates the target genes expression through cleaving mRNA and translational suppression [6]. Many evidences reported that miRNA play an important role in many biological process are cell proliferation, angiogenesis, invasion, apoptosis and organ development in different types of cancer $[7,8]$ and, are closely associated with development cell chemoresistance in cancer cells especially in colorectal cancer [9-13]. In this study, we performed identification the aberrant expression of different miRNA in the parental and Oxaliplatin-resistant CRC using microarray analysis. Further, we identified a single miRNA, miR-100-5p, which was most significantly upregulated in Oxaliplatin-resistant CRC cells. Previous study reported that mir-100-5p might be has function as an oncogenic role [14] or a tumor suppressor [15] for many type of cancers. Interestingly, some report suggest that miR-100-5p displaying role in anticancer effects for chemotherapeutic drugs in multiple human cancers [16]. However, the detailed molecular mechanisms of miR-100-5p gene involved in regulation Oxaliplatin drug resistance in CRC cell needs to clarified. Chemoresistant is the alteration responses of cell cycle towards chemotherapy agents in tumor cells. CTDSPL gene, is belongs to the C-small terminal domain phosphate family which located at 3p21.3, by removing phosphate group from Rb1 serine on ser-807 and ser-811. Previous studies reported that CTDSPL regulates the signaling pathway of $\mathrm{p}-\mathrm{RB} / \mathrm{E} 2 \mathrm{~F} 1$ and induces the cell cycle arrest at the G1/S boundary. miR-181 family modulates myeloid differentiation in both uveal melanoma and acute myeloid leukemia cells through targeting CTDSPL $[17,18]$. Further, CTDSPL partly involved in the regulating cell growth in CRC still needs to study.

Here, we investigate that expression of mir-100-5p enhanced chemoresistance in CRC cell through suppressing CTDSPL expression and subsequently modulates the CTDSPL/p-RB/E2F1 signaling pathway which is involve in overcoming CRC chemoresistance to Oxaliplatin drug.

\section{Materials And Methods}

\subsection{Cell culture, antibodies and reagents}

LoVo colorectal cancer cell (CRC) line is derived from Bioresource Collection and Research Center (Hsinchu, Taiwan). CRC cells were mantained in Dulbecco's Modified Eagle's Medium-low glucose (D5523-1L, Sigma Aldrich, Missouri, USA) supplemented with 10\% fetal bovine serum (Gibco Life Technologies Co., Grand Island, NY, USA), 1\% penicillin/streptomycin, sodium bicarbonate at $37^{\circ} \mathrm{C}$, with $5 \% \mathrm{CO}_{2}$ with $80-90 \%$ humidified condition. The cell medium was changed after $48 \mathrm{~h}$ subculture. Oxaliplatin powder and 1-(4, 5-Dimethylthiazol-2-yl)-3, 5-diphenylformazan (MTT) was purchased from SigmaAldrich (Sigma Chemical Co., Merck KGaA, Darmstadt, Germany). followed the antibodies that we used 
are CTDSPL (Novus Biologicals Co., Centennial CO, USA); E2F1, phosphorylated RB and RB (Cell Signaling Technology, Inc. Beverly, MA, USA); cyclin D1, cyclin E1, cyclin B1, cyclin A1, PCNA, ERK1/2, phosphorylated-ERK1/2, AKT, phosphorylated-AKT, p53, phosphorylated-p53, p19, p27, p15/16, Ki67 and GAPDH (Santa Cruz Biotechnology, Inc. Santa Cruz, California, USA). Secondary antibodies are HRP conjugated-Goat anti-mouse and anti-Rabbit IgG antibodies was purchased from Santa Cruz Biotechnology, Inc. (Dallas, TX, USA).

\subsection{Establishment of Oxaliplatin-resistant LoVo cancer cells}

Generating stable Oxaliplatin resistant colorectal cancer cells, first, we exposed Oxaliplatin drug with dose dependent manner $(0.0$ to $30.0 \mu \mathrm{g} / \mathrm{ml})$ in parental LoVo cancer cell for $24 \mathrm{~h}$; resulted in greater than $50 \%$ cells death (IC50 of Oxaliplatin, $15.0 \mu \mathrm{g} / \mathrm{ml}$ ). Further, added $15.0 \mu \mathrm{g} / \mathrm{ml}$ Oxaliplatin in the parental LoVo colon cancer cells $(24 \mathrm{~h}$ ) untill reached $80 \%$ confluence, and passaged twice in this same concentration $(15.0 \mu \mathrm{g} / \mathrm{ml})$ of Oxaliplatin. The repetition procedure was used, and increased doses of Oxaliplatin (20.0 and $25.0 \mu \mathrm{g} / \mathrm{ml}$ ). Next, selected the cell population that demonstrated at least a 3-fold greater IC50 (45 $\mu \mathrm{g} / \mathrm{ml})$ to Oxaliplatin than the parental LoVo cell lines.

\subsection{Microarray analysis}

RNA was isolated from the LoVo ${ }^{W T}$ and LoVo ${ }^{\text {OXR }}$ cell lines using an RNA MiniPrep kit (Zymo Research Corporation, Irvine, CA, USA) followed by the manufacturer's instructions. The microRNA expression profile was analyzed through miRNA Microarray Services (Service Code: 2h213102401; Human miRNA OneArray $\left.{ }^{\circledR}\right)$. The fold change was calculated by comparing the expression level of different miRNAs in the LoVo ${ }^{\mathrm{OXR}}$ cells as compared that LoVoWT using a $\log ^{2}$ format.

\subsection{RNA isolation and quantitative reverse transcription PCR (RT-qPCR) assay}

For Real Time-quantitative polymerase chain reaction (RT-qPCR) assay, the RNA was collected from the parental and Oxaliplatin-resistant LoVo cells using GeneJET RNA purification (Thermo Fisher Scientific, Lithuania) and then reverse transcribed to cDNA using an GScript First-Strand Synthesis Kit, cDNA synthesis kit, Oligo-dT primers $50 \mathrm{uM}$ and $10 \mathrm{mM}$ dNTP mix according to manufacturer's protocol. For examining miRNA expression level, we used a Applied Biosystem Real-Time PCR and ORA ${ }^{\text {TM }}$ SEE qPCR Green ROX L Mix, 2X (HighQu professionally simple, USA) with a total reaction volume of $20 \mu \mathrm{L}$ containing $10 \mu \mathrm{L}$ ORA ${ }^{\mathrm{TM}}$ SEE qPCR Mix, $300 \mathrm{nM}$ (each) forward and reverse primers, and $500 \mathrm{ng}$ cDNA. The primers for mir-100-5p: AACCCGUAGAUCCGAACUUGUG. mRQ 3' Primer (10 $\mu \mathrm{M})$, U6 Forward Primer $(10 \mu \mathrm{M})$, and U6 Reverse Primer $(10 \mu \mathrm{M})$ were from the Mir-X miRNA First-Strand Synthesis Kit and SYBR qRT-PCR user manual. U6 was used as an endogenous control. The gene expression was determined by

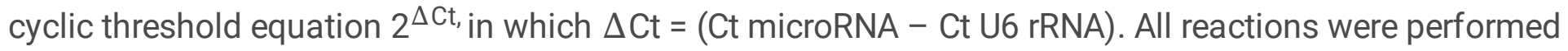
in triplicate.The primer for upstream target are GATA-1; forward: CAC TGA GCT TGC CAC ATC C, Reverse: ATG GAG CCT CTG GGG ATT A, CEBP-b; forward: CGC TTA CCT CGG CTA CCA, Reverse: ACG AGG AGG ACG TGG AGA G, FOXP3; forward: CTT CCT TGA ACC CCA TGC, Reverese: GGA GGA GTG CCT GTA AGT 
GG and the downstream target CTDSPL; Forward: AAC CCC AAG GAG GAC GAG, Reverse: CAG AAG AAG GAG CTA AGG ATG C. GAPDH; forward: GCA CCG TCA AGG CTG AGA AC, Reverse: ATG GTG GTG AAG ACG CCA GT was used as endegoneus control.

\section{5 mRNA prediction and analysis}

Three common miRNAs predictor target and binding sites algorithms that are publically available: http://www.microrna.org for miRanda algorithm, http://www.targetscan.org for TargetScan algorithm and http://mirtar.mbc.nctu.edu.tw/human/ for miRTar base algorithm were used to predict the putative targets of miR-100-5p. Integrated function and pathway analysis were performed using DAVID bioinformatics resources (http://david.abcc.ncifcrf.gov/) and significant features of predicted miRNA targets were clustered.

\subsection{Transfection}

miR-100-5p Overexpression (mimic), Knockdown (inhibitor), scrambled miRNA (mimic NC or inhibitor NC) and sh-RNA were used to transfected into cells with confluent about 60-80\%. jetPRIME®(Polyplus Transfection Inc, Illkirch, France) Kit transfection was used, the transfected cell were incubated in $5 \% \mathrm{CO}_{2}$ at $37^{\circ} \mathrm{C}$ humidified condition for $24 \mathrm{hr}$, then used in further experimental.

\subsection{Protein calculation}

Protein derived from cell extract which was using the Bradford reagent (BIO-RAD, Hercules, CA, USA) to calculate protein estimation according to the manufacturer's instruction using bovine serum albumin (BSA) as a standard $(2.0 \mathrm{mg} / \mathrm{ml})$. In brief, the calculation standard curve prepare with a range of protein concentrations $(0.0,0.1,0.2,0.3,0.4$ and $0.5 \mathrm{mg} / \mathrm{ml})$ from diluted BSA $(0.5 \mathrm{mg} / \mathrm{ml}$ final concentration). $198 \mu \mathrm{l}$ Bradford reagent was mixed with $2.0 \mu \mathrm{l}$ of protein samples in a 96-wells plate. Next, measuring the optical density (OD) of sample was at 595nm using a multiwall ELISA plate reader (Molecular Devices, Palo Alto, CA, USA) and calculated the protein amounts with previously performed BSA standard curve.

\subsection{Western blotting}

Followed the method of Huang, 2014 [19] with minor modifications, we examined the protein expression. In brief, at 14,000 rpm for 15-20 min the protein was centrifuged and 45.0 $\mu \mathrm{g}$ protein with equal amount was boiled (95 $₫ \mathrm{C}, 10 \mathrm{~min}$ ) together with appropriate $5 \mathrm{x}$ sample buffer $[10 \% \mathrm{SDS}, 50 \%$ Glycerol, $0.1 \%$ Bromophenol blue, 250mM Tris-Hcl (pH-6.8) and 5\% $\beta$-mercaptoethanol], and then loaded on 10\%-12\% sodium dodecyl sulfate-polyacrylamide gels (SDS-PAGE) at 80-100V in certain time. Next, transferred proteins on polyvinylene difluoride membrane (PVDF) (Millipore Corporation limited, Bedford, MA, USA) in transfer buffer (25mM Tris base, $190 \mathrm{mM}$ glycine and 20\% methanol) using a BIO-RAD transfer system (Bio-Rad Laboratories, Munich, Germany). Incubated protein in membrane with blocking buffer [5\% (w/v) BSA, 1xTransfer Buffer Saline (TBS) and 0.1\% Tween-20] for $1 \mathrm{~h}$ at RT. Hiring primary antibodies (1/1000$1 / 2000$ dilution in 1xTBST) at $4 \mathbb{}$ C for overnight. Thereafter, washed membrane three times with 1xTBST 
and incubated with secondary antibodies (1:4500) anti-rabbit and anti-goat HRP-secondary antibodies. Next, detection the protein signals was using custom made Immobilon ${ }^{\circledR}$ Western chemiluminescent reagent (Millipore Corporation limited, Billerica, MA, USA) on an Image Bright i500 imaging systems (Invitrogen ${ }^{\mathrm{TM}}$ iBright ${ }^{\mathrm{TM}}$ Imaging system, Thermo fisher Scientific, USA). Next, analysis the densitometry was used the ImageJ program 1.50 (National Institutes of Health, Bethesda, MD, USA). GAPDH and HDAC1 were used as endogenous control for normalization protein.

\subsection{Cell Viability Assay}

Trypan blue staining method was used to measure the cell viability and counting cell. In brief, at 15,00xg for 3-5 min trypsinized LoVo ${ }^{W T}$ and LoVo ${ }^{\text {OXR }}$ cells were centrifuged then resuspended with $1 \times$ PBS. After that, $10.0 \mu \mathrm{l}$ of cells suspension was mixed with $30.0 \mu \mathrm{l}$ of $0.3 \%$ trypan blue stained solution for $5 \mathrm{~min}$. Then, the number of colouring cells was counted using a haemocytometer under microscope. Living cell displayed colourless, while dead cells were blue colour.

\subsection{MTT [3-(4, 5-Dimethylthiazol-2-yl)-2, 5-diphenyltetrazolium-bromide] Assay}

Followed the procedure of Hsu (2019) with minor modification MTT method was used to measure anticells viability effect of cytotoxic drugs through alteration colour of MTT from light orange into a soluble purple formazon crystals using mitochondrial succinate dehydrogenase. In brief, LoVo ${ }^{\mathrm{WT}}$ and LoVo ${ }^{\mathrm{OXR}}$ cells $\left(5 \times 10^{4}\right)$ were cultivated triplicate in DMEM-Low Glucose with $10 \%$ FBS in 24 well plates for $24 \mathrm{~h}$. After that, cells were treated with increasing concentration of oxaliplatin $(0,5,10,15,20,25$ and $30 \mu \mathrm{g} / \mathrm{ml}$ ). After $24 \mathrm{~h}$ exposure, remove treated medium followed by adding medium with $100 \mu \mathrm{l}$ MTT solution $(15 \mathrm{mg} / 50 \mathrm{ml})$ then incubated at $37^{\circ} \mathrm{C}$ for $2-5 \mathrm{hr}$ until purple precipitate was formed. Thereafter, the supernatant was discard and then add $150 \mu$ l of DMSO per well for dissolving blue MTT formazon crystals. The absorbance was measured at $570 \mathrm{~nm}$ using a multi-wall ELISA plate reader (Molecular Devices, Palo Alto, $\mathrm{CA}, \mathrm{USA}$ ). $\mathrm{IC}_{50}$ wastaken as the concentration of 50 percent death of cells population.

Cell survival $($ percentage $)=\left[1-\left(O D_{\text {experiment sample }} / O D_{\text {control sample }}\right)\right] \times 100 \% .(n=3)$

\subsection{Immunocytochemistry Assay}

The cell proliferation rate of LoVoWT and LoVo ${ }^{\mathrm{OXR}}$ cancer cells was measured by immunocytochemistry assay using Ki67 staining. Briefly, LoVo ${ }^{\mathrm{WT}}$ and LoVo ${ }^{\mathrm{OXR}}$ cells were cultivated with density $5 \times 10^{3}$ cell per well of 8 well chamber slides, incubation for $24 \mathrm{~h}$. Then, fixed cells with $4 \%$ formaldehyde for $30-45$ min at room temperature. Thereafter, washed the fixed cells twice with PBS and permeabilization solution in $0.1 \%$ PBST ( $0.1 \%$ TRITON X-100 in 1XPBS solution) for $15 \mathrm{~min}$ at room temperature. Cells were blocked in blocking solution [4\% (w/v) bovine serum albumin $+0.1 \%$ Tween-20 in PBS] for $1-2 \mathrm{~h}$ at RT and incubated overnight in primary antibodies diluted with blocking solution at $4^{\circ} \mathrm{C}$, washed 3 times 5 min with $0.1 \%$ PBST, and then incubated with secondary antibodies were diluted 1:500 for fluorophores Alexa 488 at room temperature for $1-2 \mathrm{hr}$, and washed 3 times 5 min with $0.1 \%$ PBST. Coverslips were washed in distilled water prior mounting on slide with DAPI. 


\subsection{Statistical Analyses}

The analyses datas were done in three independent biological triplicates and values were represented as mean \pm standard deviation (SD). By SigmaPlot software version 11.0 (Systat Software Inc., San Jose, CA, USA) for windows with GraphPad Prism software 7.0 the mean value data were carried out. The distribution data was used one-way analysis of variance was applied followed by Student t-test. Moreover, all values were concluded as representative statistically significant with the probability was less than $0.05 \%(P<0.05)$.

\section{Results}

In this study, first we examined the effect of Oxaliplatin exposure on the cellular properties (cell viability, proliferation and EMT) of LoVo cell and LoVo Oxaliplatin-resistant colon cancer cells, and thereafter, we revealed that miR-100-5p play an oncogenic role in Oxaliplatin drug resistant CRC cells via activation of CTDSPL/pRB/E2F1 signaling pathway, promotes the cell cycle progression with suppression of cell cycle inhibitory proteins expression, and upregulate expression of Ki67, pERK1/2 and pAKT to induce cells proliferation in chemoresistant CRC cells.

\subsection{Establisment and Evaluation cell properties of parental LoVo cell and LoVo-OXR}

In the present study, we generated a stable Oxaliplatin resistant colorectal cancer cells (LoVo-OXR) by plating $1 \times 10^{6}$ LoVo cells in $10 \mathrm{~cm}$ plates and then add $15.0 \mu \mathrm{g} / \mathrm{ml}$ Oxaliplatin (IC50 of oxaliplatin) for 24 $\mathrm{h}$; resulted in $50 \%$ cells death. Following the same procedure to allowed cell growth at $80 \%$ confluence, and then passaged with increased doses of Oxaliplatin $(20.0$ and $25.0 \mu \mathrm{g} / \mathrm{ml})$, repetition procedure and allowed cell till selected at least a 3-fold greater IC50 $(45 \mu \mathrm{g} / \mathrm{ml})$ to Oxaliplatin drug resistance than the LoVo parental cell lines (Fig.1a). Next, we confirmed that the morphological feature differ between LoVo cells and LoVo-OXR cells (Fig. 1b). Furthermore, the resistance characteristics of LoVo-OXR cells was examined through MTT assay. Cell viability of LoVo-OXR cells was significant higher in variant dose of Oxaliplatin exposure for $24 \mathrm{~h}$ as compared with similarly exposed parental LoVo cells (Fig. 1c). Based on above results, we described our resistant cells as resistant to Oxaliplatin in general. Additionally, we determined the effect of chemoresistance on cell properties proliferation and epithelial-mesenchymal transition (EMT)] of parental and Oxaliplatin-resistant cells were examined by western blot and immunofluorescence analyses. The result shows that the levels of the cell proliferation expression related with proteins Ki67 (a major proliferation marker of CRC cells), p-AKT and p-ERK1/2 were highly increased in Oxaliplatin-resistant LoVo cells as compared with that parental LoVo cells (Fig.1d).

Immunofluorescence result also shows that expression of Ki67 protein was also significantly greater in the more number of cells of chemoresistant cells than in the parental cells (Fig.1e). Further, we also examined the EMT marker related with proteins E-cadherin, Vimentin, and $\beta$-catenine in LoVo cell and chemoresistant cells wherein level of all EMT marker related proteins except E-cadherin were significantly decreased in Oxaliplatin-resistant LoVo cells than in the LoVo cells (Fig. 1f). 


\subsection{Oxaliplatin-resistant LoVo cells express increased levels of miR-100-5p}

Earlier studies shown that the aberrant expression of miRNA were associated with many malignancies such as liver, leukemia and melanoma. To screen the potential role of miRNAs targets in colorectal cancer chemoresistance, we started with the analyse of miRNA expression profile in Oxaliplatin drug resistant cell lines (LoVo-OXR). The microarray platform showed that the aberrant expression level of various miRNA were different between Oxaliplatin-resistant LoVo cell and parental LoVo cell. The miR-100-5p expression as one of the observed miRNA was most significantly up regulated in LoVo-OXR cells than LoVo parental cells (Fig. 3a). According to the figure 3b, we analyze and compared the raw data of hsamiR-100-5p expression between the two CRC cell lines show that RL/C (C is LoVo cells; RL is OXR-LoVo cells) had a log2 value of $1.515 \geq 0.8,2^{\wedge \log 2}$ value of 2.85 , and $p$ value of $0.009647<0.05$ (Fig. $3 b$ ) Furthermore, we verified the microarray data of miR-100-5p expression through qRT-PCR assay wherein results confirmed that miRNA-100-5p expression was highly increased in LoVo-OXR cells as compared to LoVo cells (Fig. 3c). This results revealed that aberrant miRNA expression levels in resistant cells may associated with resistance of chemotherapy and miR-100-5p expression which has important role in the chemoresistance development of LoVo cells to Oxaliplatin.

\section{3 miR 100-5p targeting CTDSPL as a putative target in Colorectal cancer cell}

To certain putative target genes of miR-100-5p, we screened the target candidate genes using the different types of bioinformatics based miRNA target prediction data base such as TargetScan (http://www.targetscan.org/), miRanda (http://www.microrna.org/) and mirTar (http://mirtar.mbc.nctu.edu.tw/human/) (Fig. 3a). After computational analysis, we found that CTDSPL was a predicted target gene of miR-100-5p, which also known as RBS3 (RB1 serine phosphatase chromosome 3), has role major upstream mediated cell cycle progression. Knowingly, CTDSPL function as a tumor suppressor gene in many types of cancers, including uveal melanoma, acute myeloid leukemia and non-small cell lung cancer. There are one binding sequence prediction in 3 'UTR region of CTDSPL for binding to miR-100-5p and these predicted binding sequence was conserved from human (Fig. 3b). Further, western blot assay results confirmed that CTDSPL regulates downstream target gene of miR-100-5p where the level of CTDSPL was highly reduced in Oxaliplatin-resistant LoVo cell as compared with parental LoVo cells (Fig. 3c). These results conclude that CTDSPL has role as genuine key of Mir100-5p in the CRC resistant cells.

\subsection{Relationship between CTDSPL expression and cell cycle progression in chemoresistance associated Colorectal cancer cell}

Based on our finding that miR-100-5p is over expressed in Oxaliplatin-resistant CRC cell and promotes the progression cell cycle by supressing CTDSPL expression in Oxaliplatin-resistant LoVo cell. Earlier studies was reported that CTDSPL participated in regulating cell cycle progression via CTDSPL/p-RB/E2F1 pathway, through removing the phosphate group in serine 807 and 811 in its substrate, leads to form phosphorylated RB ( $\mathrm{p}-\mathrm{RB})$, further induces the release E2F1, a transcription factor, protein which leads the 
cell cycle progression (Fig. 4a). miR-100-5p could contribute to the pathogenesis of colorectal cancer via the CTDSPL/p-RB/E2F1 pathway, confirmed with Western blot assay data which indicate that the decrease expression levels of CTDSPL along with increased level of p-RB and E2F1 were found in the miR-100-5p over expression LoVo-OXR cells than LoVo-WT cells, and the level of total form RB protein remained same in both parental and Oxaliplatin-resistant LoVo Cells. It has been reported that E2F1 function as a master cell cycle regulator gene through the transcriptional regulation of dozens genes involved in DNA replication and cell cycle progression also playing an important role in cancer cell proliferation. In order to examine whether E2F1 may contribute to cell cycle progression in Oxaliplatinresistant LoVo cells, first the level of different E2F1-target cell cycle regulatory genes was evaluated through western blot assay. Interestingly, a significantly increase in the expression levels of Cdc25a, cyclin E, cyclin A, cyclin D1, PCNA, Cdk4, Cdk6 and Cdk1 but not increased expression level of cell cycle inhibitory proteins p16/p15 INK4a, p27, p-p53 and p19 were observed in Oxaliplatin-resistant LoVo cell than parental LoVo cell (Fig. 5c). Furthermore, we analyse the expression level of cyclin B1 which was unchanged between parental and Oxaliplatin-resistant LoVo cell. Infact, these results suggests that miR100-5p expression might contribute to alter cell cycle progression in Oxaliplatin-resistant CRC cell via targeting CTDSPL/p-RB/E2F1 pathway (Fig. 4b).

\subsection{Determination the upstream target of miR 100-5p in chemoresistant Colorectal cancer cell}

We extending the investigation CTDSPL as putative gene target of miR-100-5p. Next, we examine which gene regulates miR-100-5p as the upstream target through screened the bioinformatic based such as (http://alggen.1si.upc.es/cgi-bin/promo_v3) to check factor predicted within a dissimilarity margin less or equal than $15 \%$ (Fig. 5a). By checking dissimilarity index with matrix we found three candidates upstream gene target of miR-100 5p are C/EBP beta, GATA-1 and FOXP3. Previous studies showing that FOXP3 promotes the proliferation, migration, invasion in colorectal cancer cell through activation AKT signaling pathway and also involved in regulating 15-lipoxygenase-1 expression which has important role promoter in the basic transcription. The expression mRNA level of the upstream gene target of miR-100-5p are C/EBP beta, GATA-1 and FOXP3 were significantly reduced respectively when treated with variation dose of Oxaliplatin drug in LoVo-WT cells and LoVo-OXR cells were analyse used qPCR method (Fig. 5b). Meanwhile, the expression of CTDSPL was increased as we know that the Oxaliplatin drug as one of chemotheraphy colorectal cancer drug (Fig. 5c). This result is strongly evidence that CTDSPL as tumor suppressor which diplaying as gene target of miR-100-5p (Fig. 5d).

\subsection{Knockdown of upstream FOXP3 mediator regulates miR-100-5p in chemoresistant Colorectal cancer cell}

sh-RNA already known as an artificial RNA molecule which has tight hairpin that functionally as silence target gene expression through interfering RNA (RNAi). Related with our finding three transcription factor are FOXP3, CEBP-b and GATA-1 for upstream mediator which were regulating miR-100-5p expression in chemoresistant colorectal cancer cell. Next, We knockdown these three transcription factor gene of miR 100-5p in the LoVo cell (Fig. 6a). The data showed that miR-100-5p expression level was decreased 
meanwhile CTDSPL expression increased, sh-FOXP3 was involving in the regulation of miR-100-5p expression and CTDSPL in chemoresistant colorectal cancer cell. This results was convincing our finding that FOXP3 as the upstream transcription factor enhanced miR-100-5p over expression in the LoVo-OXR. On the other hand in the downstream of miR-100-5p suppressed CTDPL as a putative gene target tumor suppressor enhanced cell cycle in the chemoresistant colorectal cancer cells (Fig.6c). The expression of miR-100-5p was significant decrease when treated with sh-FOXP3 in LoVo-Oxaliplatin resistant (Fig. 6c).

\section{7 miR-100 5p overexpression downregulated CTDSPL/E2F1 and reverted upon miR-100-5p inhibition in CRC cell}

Moreover, miR-100 5p inhibition was increased the level of CTDSPL expression in chemoresistant colorectal cancer (fig.7a,b), as the convincing result that miR-100 5p enhanced chemoresistant through modulating CTDSPL/E2F1 pathway. Inhibiting miR-100 5p was downregulated of CTDSPL level which affected in cell cycle regulation presented through protein marker are Cdk4, Cdk6, Rb, CycA, E2F1 (fig.7c). Accumulating evidances have higlighted that miR-100 5p overexpression downregulated CTDSPL/E2F1 and reverted upon miR-100 $5 p$ inhibition in chemoresistant colorectal cancer.

\section{Discussion}

We revealed the oncogenic role of miR-100-5p gene for developing chemoresistant in colorectal cancer cells associated with altered cell cycle progression, reduction of apoptosis, promoting cell proliferation via regulating the CTDSPL/p-RB/E2F1 mediated signaling pathway. We found that CTDSPL, $\mathrm{p}-\mathrm{RB}$ and E2F1 level were highly increased in chemoresistant CRC cell compared with the parental cells which is associated with high proliferation rate. Moreover, CTDSPL expression was negatively correlated with the expression of miR-100-5p in drug-resistant CRC cell. Therefore, our study conclude that miR-100-5p may plays an important role for regulating the cell cycle-mediated chemoresistance in colorectal cancer cells.

Previous reports had been reported that miR-100-5p expression was very well associated with chemotherapeutic resistance of cancer cells. miR-100-5p promoted prostate cancer proliferation, and silencing of miR-100-5p gene enhanced the apoptosis and reverse castration-induced resistance cell cancer [19]. The down regulation of miR-100-5p expression is significantly decreased non-small cell lung cancer (NSCLS) proliferation, ability colony-forming and significantly enhanced sensitivity of NSCLC cells to first line of chemotherapeutic drugs (crizotinib, lorlatinib, and cisplatin) [20]. The silencing of miR-100$5 p$ was also observed to increase resensitivity of resistant epithelial ovarian cancer to Cisplatin [16]. In addition to this, up-regulation of miR-100-5p definitely decreased the proliferation, migration and colony forming ability in numerous types of cancer cells including, prostate cancer, gastric cancer, endometrial cancer, chordoma cancer and colorectal cancer [15, 21-23]. In current study, we observed that upregulation of miR-100-5p expression that analysis by microarray and qRT-PCR, significantly promoted cell proliferation and cell viability in chemoresistant CRC cell than the parental cell. Further, in CRC cell Oxaliplatin induced apoptosis was prominently decreased than parental CRC cells and its sensitivity was 
significantly increased in parental CRC cells. Therefore, increased expression of miR-100-5p might be associated with chemotherapy resistance in CRC cells.

Alteration cell cycle regulatory genes can deregulated proliferation, cells growth and cell cycle progression, which results in various types of pathological conditions including human malignancy [24, 25], in colorectal cancer (CRC) cells, Mir-6883 miRNA inhibited CRC cells proliferation, invasion, and migration, and also induces cell cycle arrest at G1-phase by targeting CDK4/6 [26]. He et al. [27] reported that Mir-218 induce apoptosis, inhibited cell proliferation and caused cell cycle arrest at G2-phase in colon cancer cells. However, Li et al. [28], reported that Mir-18b significantly promoted the CRC cells proliferation, metastasis and cell cycle progression through targeting of CDKN2B.

Our present study results showed that the cell cycle phase progression was increased in chemoresistant CRC cells than parental CRC cells. Bioinformatics analysis and western blot assay results confirmed CTDSPL was the predicted target of Mir-100-5p. CTDSPL gene, previously known as RBPS3 or HYA22, a phosphatase like tumor suppressor gene located at 3p21.3, and belongs to the small C-terminal domain phosphatase family, which remove phosphate group from serine of Rb1 on ser-807 and ser-811. CTDSPL was reported modulates the p-RB/E2F1 mediated signaling cascade and induces the cell cycle arrest at the $\mathrm{G} 1 / \mathrm{S}$ boundary $([17,23]$. Here, the association level between significant was observed decrease of CTDSPL level and increase of pRB and E2F1 level in oxaliplatin-resistant CRC cells as comparison with parental CRC cells (Fig. 8). These result is consistent with earlier studies, wherein down regulation of CTDSPL level promoted cells proliferation, myeloid differentiation and cell cycle progression (S-phase progression) in Mir-100-5p and Mir-181b expressing uveal melanoma and acute-myeloid leukemia cells, respectively $[17,23]$.

As big noted of evidence reported that the ' $R b$ ' pathway as a key role in cell physiology and different types of pathologies including malignant transformation via different molecular mechanisms are through meditation of G1-phase cycle progression and the G1/S-phase transition [25, 29-34]. Next study, we found that upregulation of pRB enhanced the disruption of pRB/E2F1 complex and, subsequently release of E2F1 which could be further increase the cell cycle progression (G1-S and S-phase transition) and cells proliferation in chemoresistant CRC cells. E2F1, a key downstream binding protein of tumor suppressor protein $\mathrm{Rb}$, can function as a master cell cycle regulator gene through the transcriptional regulation of dozens genes involved in DNA replication and cell cycle progression and also plays a critical role in cancer cell proliferation. Emerging evidences recommend that the expression of E2F1 was higher in a various types of human malignancies and it's positively associated with a high grade tumors and worse prognosis conditions [35-37]. Moreover, the cell cycle progression were positively associated by the over expression of E2F1 in different types of cancer tissues [37-40], showing that E2F1 expression might involved in the regulation cell cycle development and progression of tumors. Chang et al. [38] reported that the activation of E2F1 by FGF9 was involved in the cell cycle progression and proliferation in mouse Leydig tumor cells. Additionally, Baldini et al. [41] showed that E2F1-overexpression was positively correlated with increased cyclin-A and Ki67 expression and as a results reduced disease free survival in node-negative breast cancer patients. Further, earlier studies illustrated that the activation of E2F1 leading 
to the transcription of genes encoding cell cycle progression and DNA synthesis related proteins such as cyclin E, cyclin D, cyclin A, Cdk2, Cdk4/6 and cdc25 [38]. The cell cycle is important in regulating cell growth. The complex of cyclin and Cdks proteins can promote cell cycle progression from one phase to another phase and their deregulation during cell cycle transit normal cells to malignant cells [39].

In this study, level of cyclin D1, cyclin E1, Cdc25a, Cdk4 and Cdk6 for G1phase, Cyclin E1, Cdk2 and PCNA for S-phase, cyclin A1 and Cdk2 for S/G2 phase were increased, while level of cyclin B1 for G2/M phase was not changed, in Oxaliplatin-resistant CRC cells than the parental cells. Next, we examined the status of cell cycle inhibitor proteins in parental and chemoresistant CRC cells. p53 is a well-known cell cycle inhibitory protein and function as a tumor suppressor that can downstream regulation of p21 and p27 proteins to further induce cell cycle arrest through interacts with cyclin/Cdk complex. Here, we observed that the expression of p15, p16 and p19 for G1-phase and p-p53 and p27 for G1-S phase and S-phase inhibition were reduced in oxaliplatin-resistant CRC cells than the parental CRC cells, indicating p53 and also other cell cycle inhibition pathway not showing any role in suppressing chemoresistant CRC cells proliferation. In fact, studies have shown that cells proliferation in numerous tumor tissues is due to the inactivation of cell cycle inhibition pathway and activation of pRB-E2F1 mediated pathway. In general (non-proliferating context), $\mathrm{Rb}$ maintain in dephosphorylation state by phosphatase like family proteins and interacts with DNA-bound E2F1, inhibiting its transcription function which is necessary for the G1-S and S-phase transition. When, the pRB mediated pathway is functionally inactive in most types of cancer cells, as a result deregulation of E2F1 activity which leading to uncontrolled proliferation (Fig. 8a). Our data also showed that the expression of standard cell proliferation markers Ki67, p-AKT, and p-ERK1/2 decreased in oxaliplatin-resistant CRC cells as compared with parental cells, indicating the pRB/E2F1 mediated pathway plays a significant role in CRC cells proliferation. Many studies have shown that E2F1 dependent cells proliferation was found to be mediated by up-regulation of EGFR and activation of cytoplasmic Ras/mitogen-activated protein kinase (MAPK)/extracellular signal-regulated kinase (ERK) and PI3K/AKT signaling cascades as well as transactivation of Ki67 in vivo and in vitro [39,42-43].Thus our results was parallel to other studies.

Figure 8. Proposed mechanism of miR-100-5p enhanced cell cycle mediated chemoresistant through modulating CTDSPL/pRB/E2F1 signaling pathway in CRC. (a) miR-100-5p level is highly expressed in LoVo-OXR meanwhile CTDSPL expression is downregulated. (b) CTDSPL is downstream putative target of miR-100-5p and FOXP3 is upstream target of miR-100-5p in chemoresistant colorectal cancer cell.

Additionaly, miR-100-5p inhibitor was used to confirmed that CTDSPL expression was upregulated which leads to upregulated the apoptotic and deactivated cell proliferation. To extend our finding, we were checking the upstream target of miR-100-5p. Moreover, in qRT-PCR result showed that among of the upstream, FOXP3 was significantly upregulated in miR-100-5p and suppressed CTDSPL expression. The other hand, shRNAi of FOXP3 was downregulated the expression of miR-100-5p and upregulated the CTDSPL expression in LoVo-OXR and LoVo-WT (Fig. 8b). So, we concluded that FOXP3 as mediator of mir-100-5p regulation in chemoresitant colorectal cancer cell lines. Supportedly with previous reports that the expression of FOXP3 in colorectal cancer cell correlates with disease progression in patients with 
colorectal cancer [47], also as a marker of CD $4+C D 25$ + regulatory T cells which is determinant of their immunosuppressive functions and shown limit antitumor immune responses during tumor progression [48]. FOXP3 has important role in the development of colorectal cancer disease.

In summary, we recommend that mir-100-5p plays an oncogenic role in oxaliplatin-resistant CRC cells via activation of CTDSPL/pRB/E2F1 signaling pathway through reduced CTDSPL expression, promotes the cell cycle progression with suppression of cell cycle inhibitory proteins expression, and upregulate expression of Ki67, pERK1/2 and pAKT to induce cells proliferation in chemoresistant CRC cells. In addition our finding of upstream target miR-100-5p is FOXP3, mediating the regulation of miR-100-5p in chemoresistant clorectal cancer which is also affected on CTDSPL expression in CRC cells.

\section{Conclusions}

In this study, we found the novel finding that increased expression of miR-100-5p contributes to alter cell cycle progression through the reducing the expression of CTDSPL, which in turn dephosphorylates of RB and release the downstream effector E2F1 and promotes the cell cycle progression in Oxaliplatinresistant CRC cells. Furthermore, miR-100-5p function as an oncogenic gene in chemoresistant CRC cells through predicted bind to the 3'-UTR of CTDSPL and inhibits its expression in the Oxaliplatin-resistant LoVo cells. Taken together, this study also suggests that an increased expression of miR-100-5p may presented an oncogenic role in chemoresistant colorectal cancer cells through disrupting the cell cycle control, reduction of apoptosis, promoting cell proliferation and consequently enhancing the development of chemoresistant conditions in LoVo cells via regulating CTDSPL/p-RB/E2F1 pathway. In order for convincing our finding, we also checked the upstream target of miR-100-5p that is FOXP3, as mediator of miR-100-5p regulation in CRC cell. In line with expectation shRNAi FOXP3 was downregulated the expression of miR-100-5p, in turn upregulated the expression of miR-100-5p. Further studies to identify the therapeutic role of Mir-100-5p in the pathologies of CRC cells in animal model is needed. The manipulation of Mir-100-5p could be diagnostically and therapeutically relevant for the treatment of colorectal tumor patients with chemoresistance.

\section{Abbreviations}

\begin{tabular}{|ll|}
\hline miR-100-5p & MicroRNA -100-5 \\
\hline LoVo-OXAR & LoVo-Oxaliplatin Resistant cell line \\
\hline CRC & Colorectal Cancer \\
\hline CTDSPL & Carboxy-Terminal Domain Small Phosphatase Like \\
\hline FOXP3 & Transcription Factor Forkhead box \\
\hline E2F1 & Transcription factor E2F1 \\
\hline Rb/p-Rb & Retinoblstoma protein \\
\hline
\end{tabular}




\section{Declarations}

Author's constribution: Ming-Cheng Chen: Conceptualization, Data curation, Formal analysis, Investigation, Conceptualization, Writing-review \& editing. Hema Sri Devi, Chaouhan Hitesh Singh: Data curation, Formal analysis, Writing-original draft, Investigation, Methodology, review \& editing. Tso-Fu Wang: Conceptualization, Data curation, Formal analysis, Visualization, Software. Chi-Cheng Li: Project administration, Resources, Software. Tsung-Jung Ho, Tzu-Ching Shih: Supervision, Validation, Visualization. Marthandam Asokan Shibu: Supervision, Validation, Visualization. Wei-Wen Kuo: Project administration, Resources, Software. Yu-Jung Lin: Methodology, Project administration, Resources, Supervision, Funding acquisition. Chih-Yang Huang: Conceptualization, Data curation, Funding acquisition, Investigation, administration, Resources, Supervision, Validation. All authors revised the manuscript critically for important intellectual content and approved the final version of the manuscript.

Conflicts of Interest : All authors declare that there is no any competing interest exists among them.

Consent to parcitipate : Not applicable.

Consent for publication: Not applicable.

Funding : This study was supported by China Medical University Hospital, Departement Medical Research (DMR-110-209) and Ministry Of Science and Technology, Taiwan (MOST :109-2320-B-303-001-MY2)

\section{References}

1. D. Cunningham, W. Atkin, H.J. Lenz, H.T. Lynch, B. Minsky, B.Noerdlinger, N. Starling, Colorectal cancer. Lancet. 375(9719):1030-1047.(2010)

2. L. Fang, H. Li, H. Wang, J. Hu, T. Jin, J. Wang, B.B Yang, MicroRNA-17-5p promotes chemotherapeutic drug resistance and tumor metastasis of colorectal cancer by repressing PTEN expression, Oncotarget.5(10): 2974-87. (2014)

3. P.M. Kasi, A Grothey, Chemotheraphy maintenance. Cancer journal. 22(3):199-204.(2016)

4. C. Cremolini, M. Schirripa, C. Antoniotti, R. Moretto, L. Salvatore, G. Masi, A. Falcone, F. Loupakis, First-Line chemotheraphy for $\mathrm{mCRC}$-a review and evidance-based algorithm. Nature Reviews. Clinicla Oncology.12(10): 607-619. (2015)

5. M. Hewish, D. Cunningham, First-line treatment of advanced colorectal cancer. Lancet. 377(9738): 2060-2062.(2011)

6. G.Hutvagner, P.D. Zamore, A microRNA in a multiple-turnover RNAi enzyme complex. Science. 297(5589): 2056-2060.(2002)

7. D.P. Bartel, MicRNAs: genomics, biogenesis, mechanism, and function, Cell. 116(2): 281-297. (2004)

8. K. Kai, R.L. Dittmar, S. Sen, Secretory microRNAs as biomarkers of cancer, Seminar in Cell and Developmental Biology. 78: 22-36. (2018) 
9. B. Song, Y. Wang, M.A. Titmus, G. Botchkina, A. Formnetini, M. Kornmann, J. Ju, Molecular mechanism of chemoresistance by miR-215 in osteosarcoma and colon cancer cells, Molecular Cancer. 9:96.(2010)

10. B. Song, Y. Wang, Y. Xi, K. Kudo, S, Bruheim, G.I Botchkina, E. Gavin, Y. Wan, A. Formentini, A. Kornmann, M. Fodstad, O. Ju, J. Ju, Mechanism of chemoresistance mediated by miR-140 in human osteosarcoma and colon cancer cells, Oncogene. 28(46): 4065-74.(2009)

11. Z. Xiao, S. Chen, S.Feng, Y. Li, J. Zou, H, Ling, Y. Zeng, X. Zeng, Function and mechanisms of microRNA-20a in colorectal cancer (Review), Experimental and therapeutic medicines, 19(3): 16051616.(2020)

12. T. Jiang, L. Ye, Z. Han, Y. Liu, Y. Yang, Z. Peng, J. Fan, miR-19b-3p promotes colon cancer proliferation and oxaliplatin-based chemoresistance by targeting SMAD4: Validation by informatics and experimental analyses Journal of Experimental \& Clinical Cancer Research. 36(1): 131.(2017)

13. H. Liang, Y. Xu, Q. Zhang, Y. Yang, Y. Mou, Y. Gao, R. Chen, C. Chen, P. Dai, MiR-483-3p regulates oxaliplatin resistance by targeting FAM171B in human colorectal cancer cells, Artificial cells, nanomedicine \& Biotechnology, 47(1): 756-736, (2018)

14. K. Takebayashi, K. Nasu, M. Okamoto, Y. Aoyagi, T. Hirakawa, H. Narahara, hsa-miR-100-5p, an overexpressed miRNA in human ovarian endometriotic stromal cells, promotes invasion through attenuation of SMARCD1 expression, Reproductive Biology and Endocrinology, 18:31. (2020)

15. S.L. Li, Y. Ye, J.J Wang, MiR-100-5p downregulates mTOR to suppress the proliferation, migration and invasion of prostate cancer cells, Cold Spring Harbor Laboratory Press. (2020).doi: https://doi.org/10.1101/2020.07.30.228676.

16. P. Guo, X. Xiong, S. Zhang, D. Peng, miR-100 resensitizes resistant epithelial ovarian cancer to cisplatin, Oncology reports. 36(6):3552-3558. (2016)

17. Y.S. Zheng, H. Zhang, X.J. Zhang, D.D. Feng, X.Q. Luo, C.W. Zeng, K.Y. Lin, H. Zhou, L.H. Qu, P. Zhang, Y.Q. Chen, MiR-100 regulates cell differentiation and survival by targeting RBSP3, a phosphatase-like tumor suppressor in acute myeloid leukemia. Oncogene, 31(1): 80-92.(2012)

18. H.H. Hsu, W.W. Kuo, H.N.Shih, S.F. Cheng, C.K. Yang, M.C. Chen, C.Y. Huang, FOXC1 regulation of miR31-5p Confers Oxaliplatin Resistance by Targeting LATS2 in Colorectal Cancer, Cancer. 11:1576. (2019)

19. N.Nabavi, N.Saidy, E.Venalainen, A. Haegert, A. Parolia, H. Xue, Y. Wang, R. Wu, X. Dong, C. Collins, F. Crea, F. Wang, miR-100-5p inhibition induces apoptosis in dormant prostate cancer cells and prevents the emergence of castration-resistant prostate cancer, Scientific reports. 7(1):4079.(2017)

20. Y. Lai, M. Kacal, M. Kanony, I. Stukan, K. Jatta, L. Kis, E. Norberg, H. Vakifahmetoglu-Norberg, R. Lewensohn, P. Hydbring, S. Ekman, miR-100-5p confers resistance to ALK tyrosine kinase inhibitors Crizotinib and Lorlatinib in EML4-ALK positive NSCLC, Biochemical and Biophysics research Communications. 511(2):260-265.(2019)

21. D.B. Shi, Y.W. Wang, A.Y. Xing, J.W. Gao, H. Zhang, X.Y. Guo, P. Gao, C/EBPa-induced miR-100 expression suppresses tumor metastasis and growth by targeting ZBTB7A in gastric cancer, Cancer 
letters. 369(2):376-385. (2015)

22. J. Cai, Y. Zhang, S. Huang, M, Yan, J. Li, T. Jin, S. Bao, MiR-100-5p, Mir-199a-3p and Mir-199b-5p induce autophagic death of endometrial carcinoma cell through targeting mTOR, International Journal of Clinical and Experimental Pathology. 10(9): 9262-9272.(2017)

23. L.Zhang, X. He, F. Li, H. Pan, X. Huang. X. Wen, H. Zhang, B. Li, S. Ge, X. Xu, R. Jia, X. Fan, The MiR181 family promotes cell cycle by targeting CTDSPL, a phosphatase-like tumor suppressor in uveal melonama, Journal of clinical and experimental research. 37(1):15.(2018)

24. T. Hunter, J. Pines, Cyclins and cancer II: Cyclin D and CDK inhibitors come of age, Cell. 79(4): 573582. (1994)

25. C.J. Sherr, Cancer cell cycles, Science. 274(5293):1672-1677.(1996)

26. A.R. Lulla, M.J. Slifker, Y. Zhou, A. Lev, M.B. Einarson, T.D. Dicker, W.S. El-Deiry, miR-6883 family miRNAs Target CDK4/6 to induce G1 Phase Cell-Cycle Arrest in Colon Cancer Cells, Cancer Research. 77(24):6902-6913.(2013)

27. X. He, Y. Dong, C.W. Wah, Z. Zhao, S.S.M. Ng, F.K.L. Chan, J.J.Y. Sung, J. Yu, MicroRNA-218 inhibits cell cycle progression and promotes apoptosis in colon cancer by downpolycombegulating BMI1 polycomb ring finger oncogene, Molecular Medicine. 18(1): 1491-1498.(2013)

28. Y. Li, M. Chen, J. Liu, L. Li, X. Yang, J. Zhao, M. Wu, M. Ye, Upregulation of MicroRNA 18b Contributes to the Development of Colorectal Cancer by Inhibiting CDKN2B, Molecular and Cellular Biology, 37(22):e00391-17.(2017)

29. P.L. Chen, P. Scully, J.Y. Shew, J.Y. Wang, W.H. Lee, Phosphorylation of the retinoblastoma gene product is modulated during the cell cycle and cellular differentiation, Cell. 58(6):1193-1198(1989)

30. M. Strauss, J. Lukas, J. Bartek, Unrestricted cell cycling and cancer, Nature pMedicine.1(12):12451246.(1995)

31. R.A. Weinberg, The Retinoblastoma protein and cell cycle control, Cell. 81(3):323-330.(1995)

32. J. Bartek, J. Bartkova, J. Lukas, The Retinoblastoma protein pathway and the restriction point, Current Opinion in Cell Biology. 8(6):805-814.(1996)

33. J. Bartek, J. Bartkova, J. Lukas, The retinoblastoma protein pathway in cell cycle control and cancer, Experimental Cell Research. 237(1):67-108.(1997)

34. M. Hall, G. Peters, Genetic alterations of cyclins, cyclin-dependent kinases and Cdk inhibitors in human cancer, Advances in Cancer Research, 68:67-108.(1996)

35. P.K. Tsantoulis, V.G. Gorgoulis, Involvement of E2F transcription factor family in cancer, European Journal of Cancer. 41(16):2403-14.(2005)

36. P.J. laquinta, J.A. Lees, Life and death decisions by the E2F transcription factors, Current Opinion in Cell Biology.19(6):649-57.(2007)

37. L.N. Kent, G. Leone, The broken cycle: E2F dysfunction in cancer, Nature Reviews Cancer. 19(6):326338. (2019) 
38. M.G.J. Navarro, C. Basset, T. Arcondeguy, C. Touriol, G. Perez. H. Prats, E. Lacazette, Api5 Contributes to E2F1 control of the G1/S Cell cycle Phase Transition. Plos One. 8(8):e71443.(2013)

39. M.M. Chang, M.S. Lai, S.Y. Hong, B.S. Pan, H. Huang, H.S. Yang, C.C. Wu, H.S. Sun, J.I. Wang, C.Y. Huang, FGF9/FGFR2 increase cell proliferation by activating ERK1/2. Rb/E2F1 and cell cycle pathways in mouse Leydig tumor cells, Cancer Science. 109(11):3503-3518.(2018)

40. L.A. Sheldon, Inhibition of E2F1 activity and cell cycle progression by arsenic via retinoblastoma protein, Cell cycle.16(21):2058-2072. (2017)

41. E.Baldini, A. Camerini, A. Sgambato, T. Prochilo, A. Capodanno, F. Pasqualetti, C. Orlandini, L. Resta, L. Bevilacqua, L. Collecchi, Cyclin A and E2F1 Overexpression correlate with reduced disease-free survival in node-negative breast cancer patients, Anticancer Research, 26(6B):4415-21(2006)

42. V. Alla, D. Engelmann, A. Niemetz, J. Pahnke, A. Schmidt, M. Kunz, S. Emmrich, M. Steder, D. Koczan, B.M.Pützer, E2F1 in melanoma progression and metastasis, Journal of the National Cancer Institute. 102(2):127-33.(2010)

43. D.Engelmann, B.M. Pützer, The dark side of E2F1: in transit beyond apoptosis, Cancer Research, 72(3):571-575.(2012)

44. C.C. Lin, K.B. Chen, C.H. Tsai, F.J. Tsai, C.Y. Huang. C.H. Tang, J.S. Yang, Y.M. Hsu, S.F. Peng, J.G. Chung. Castilian inhibits human prostate cancer DU 145 cell migration and invasion via Ras/Akt/NFB signaling pathways. Journal of Food Biochemistry. 43(7):e12902.(2019)

45. S.P. Liu, M.A. Shibu, F.J. Tsai, Y.M. Hsu, C.H. Tsai, J.G. Chung, J.S. Yang, C.H. Tang, S. Wang, Q. Li, C.Y. Huang, Tetramethylpyrazine reverses high-glucose induced hypoxic effects by negatively regulating HIF-1 alpha induced BNIP3 expression to ameliorate H9c2 cardiomyoblast apoptosis, Nutr Metab (Lond). 17:12.(2020)

46. W.S. Chang, C.W. Tsai, J.S. Yang, Y.M. Hsu, L.C. Shih, H.Y. Chiu, D.T. Bau, F.J. Tsai, Resveratrol inhibited the metastatic behaviors of cisplatin-resistant human oral cancer cells via phophorylation of ERK/p-38 and supression of MMP-2/9, J Food Biochem. 45(6):e13666. (2021)

47. M.Kim, T. Grimmig, M. Grimm, M. Lazariotou, E. Meier, A. Rosenwald, I. Tsaur, R. Blaheta, U.Heemann, C.T. Germer, A.M.W, Gasser, M. Gasser, Expression of FOXP3 in Colorectal Cancer but Not in Treg Cells Correlates with Disease Progression in Patients with colorectal cancer, PLoS ONE 8(1): e53630. (2013)

48. T. Grimmig, M. Kim, C.T. Germer, M. Gasser, A.M.W. Gasser, The role of FOXP3 in disease progression in colorectal cancer patients,Oncolmmunology. 2:e24521. (2013)

\section{Figures}


Figure 1

(A)

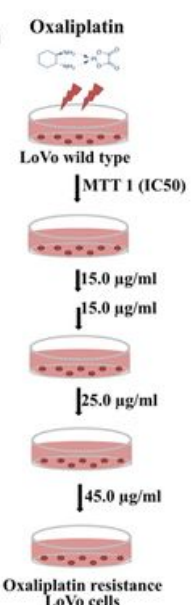

LoValiplatin resistane
Lovo cells
$\mathrm{LOVo}^{\text {WT }}$ cells $\quad \mathrm{LoV}_{0}{ }^{\text {OXR }}$ cells

(B)
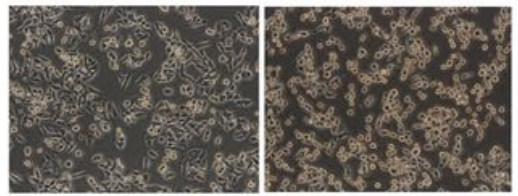

(C)
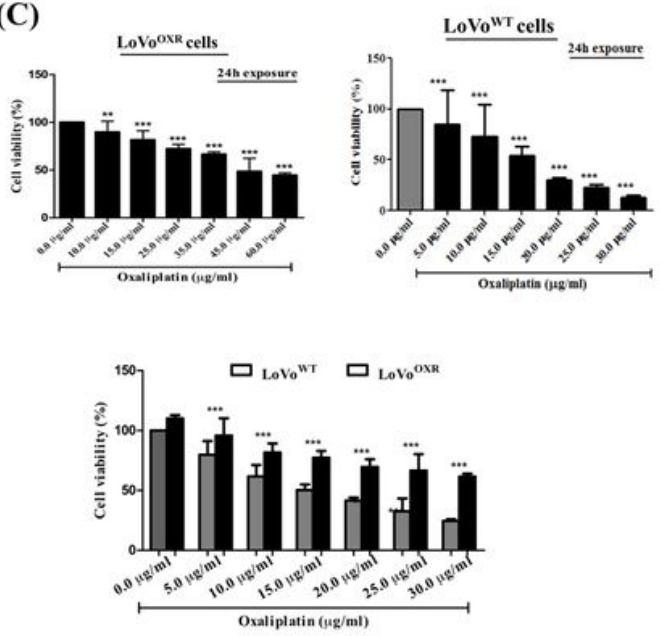

(D)

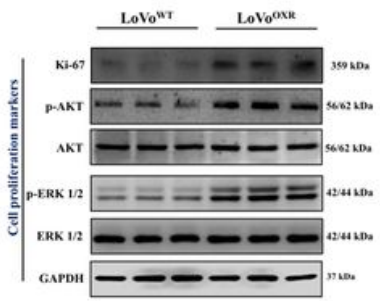

(E)

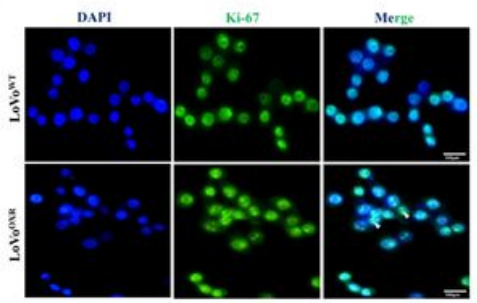

(F)

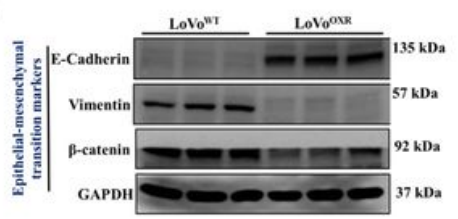

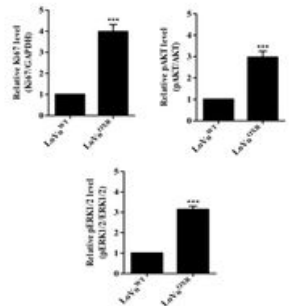

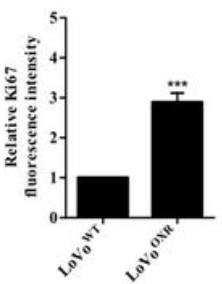

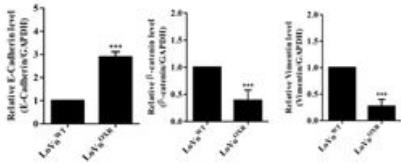

\section{Figure 1}

Generating and evaluation cell properties of parental LoVo cell and Oxaliplatin-resistant LoVo cell lines.

(a) Establishment Oxaliplatin-resistant LoVo cell lines and IC50 of Oxaliplatin. (b) The morphology of LoVo parental and LoVo-OXR. (c) Both LoVo parental and LoVo-OXR cell lines were treated with varying concentration of Oxaliplatin for $24 \mathrm{hr}$ analyzed cell viability. (d) Cell properties altered between parental and Oxaliplatin resistant (OXR)-LoVo cells. Western blot analysis of the expression levels of the cell proliferation marker (Ki67, p-AKT and p-ERK1/2) in parental and Oxaliplatin-resistant LoVo cells. (e) The Immunofluorescence images showed that the expression level of Ki67 was greater in the more number of cells of chemoresistant cells than in the parental cells. (f) Western blot analysis showed that the expression level of all EMT marker related proteins except E-cadherin were significantly decrease in chemoresistant cells than in parental cell. All protein expression normalized against to GAPDH used as a loading control. Quantification of the data was carried out by ImageJ software 1.50 and are presented as the mean $\pm S D(n=3)$. Significance ascribed as * $p<0.05$ vs. Oxaliplatin-resistant LoVo cells. 
Figure 2

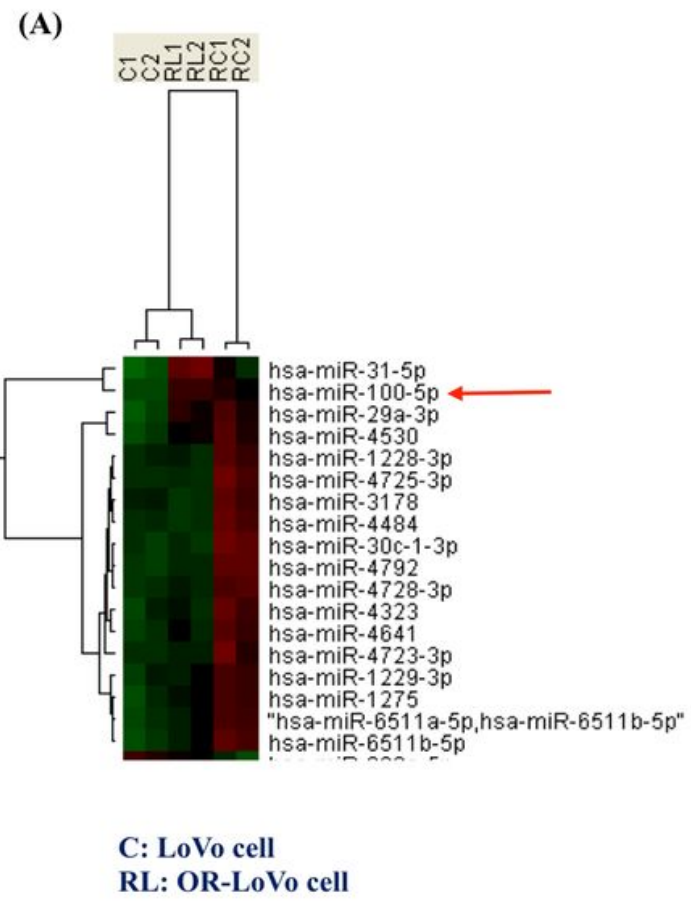

(B)

\begin{tabular}{|l|l|l|}
\hline \multicolumn{2}{|c|}{ Name } & \multicolumn{1}{c|}{ hsa-mir-100-5p } \\
\hline \multirow{2}{*}{$\begin{array}{l}\text { Normalized } \\
\text { intensity }\end{array}$} & $\mathrm{C}$ & 93.0 \\
\cline { 2 - 3 } & $\mathrm{RL}$ & 185.25 \\
\hline $\mathrm{CV}$ & $\mathrm{C}$ & 0.000 \\
\cline { 2 - 3 } & $\mathrm{RL}$ & 0.002 \\
\hline Log2 (ratio) & $\mathrm{RL} / \mathrm{C}$ & 0.9942 \\
\hline $2^{\wedge} \log 2$ & $\mathrm{RL} / \mathrm{C}$ & 1.991 \\
\hline P-value & $\mathrm{RL} / \mathrm{C}$ & 0.001725 \\
\hline
\end{tabular}

(C)

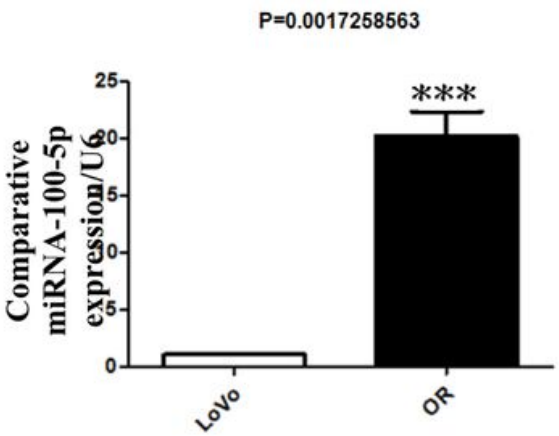

\section{Figure 2}

\section{MicroRNA expression in LoVo and Oxaliplatin-resistant LoVo cell lines.}

(a) MiRNA microarray data analysis with the red bar indicating upregulated expression and the green bar indicating downregulated expression. (b) Detailed miRNA microarray data analysis lists the hsa-miR-100$5 p$ C, RL, or RL/C (C RL is OR-LoVo cells) value. C is LoVo cells; RL is OR-LoVo cells. (c) Results of the qRT- PCR the expression levels of miR-100-5p are shown by the bar. ${ }^{\star \star \star} p<0.001$ vs. LoVo cells. 


\section{Figure 3}

(A)

\begin{tabular}{|c|c|}
\hline Target genes & hsa-miR-100-5p \\
\hline miRTarBase & $\mathbf{2 4 6}$ \\
\hline miRDB & $\mathbf{2 7}$ \\
\hline Targetscan & 39 \\
\hline $\begin{array}{c}\text { Combined } \\
\text { (miRTarBase, Targetscan } \\
\text { \&miRDB) }\end{array}$ & 6 \\
\hline
\end{tabular}

(C)

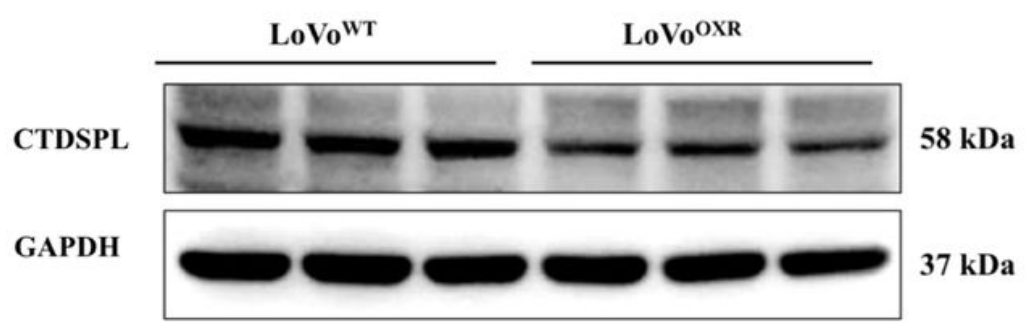

hsa-miR-100-5p

Both (miRTarBase \&miRDB)

\begin{tabular}{|c|c|c|}
\hline KBTBD8 & mTOR & FGFR3 \\
\hline HS3ST2 & SMARCA5 & CTDSPL \\
\hline
\end{tabular}

(B)

\begin{tabular}{|c|c|c|c|c|c|c|}
\hline & & 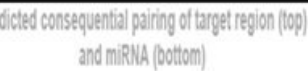 & Site & $\begin{array}{l}\text { Contexity } \\
\text { scover }\end{array}$ & $\begin{array}{l}\text { Contextit score } \\
\text { percentle }\end{array}$ & $\begin{array}{l}\text { Weighted } \\
\text { contextits score }\end{array}$ \\
\hline Postion 25652.256 & $25^{\prime}$ & 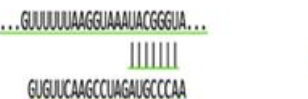 & 8 mer & 0.71 & 99 & .053 \\
\hline
\end{tabular}

Figure 3

\section{CTDSPL as a putative target of miR-100-5p in Colorectal cancer cells.}

(a) We used three computational algorithms (miRTarBase, miRDB, and TargetScan Human) databases to predict the putative targets of has-miR-100-5p, and found that 6 common predicted targets from the above mentioned three online databases. (b) Image showing the putative target binding sites of CTDSPL to Mir-100-5p. (c) Western blot analysis used to confirm the expression level of CTDSPL in the two cell lines. GAPDH was used as a loading control. Quantification of data was carried out by ImageJ software 1.50 and are presented as the mean $\pm S D(n=3)$. Significance ascribed as $* p<0.05$ vs. Oxaliplatin-resistant LoVo cells. 
Figure 4

(A)
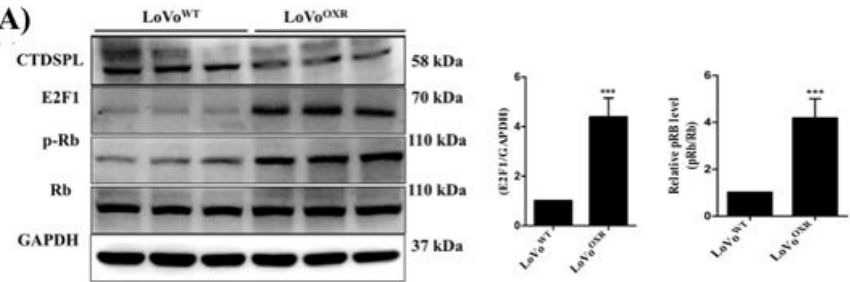

(C)
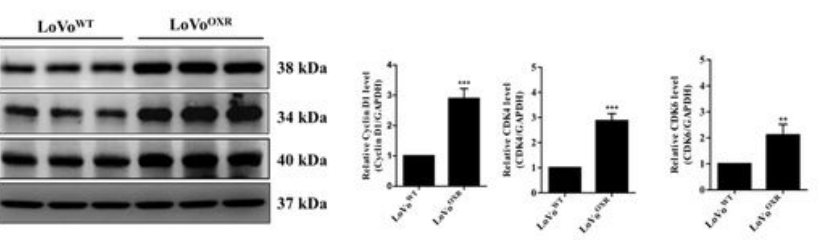

(D)
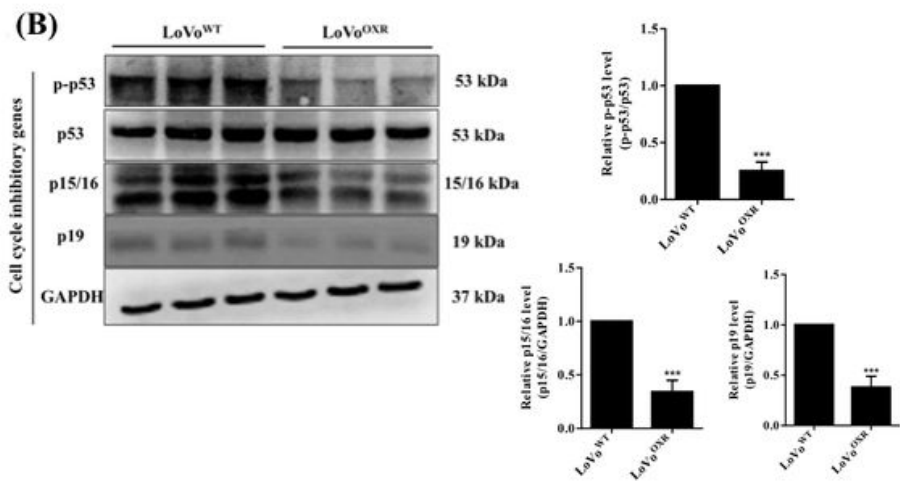

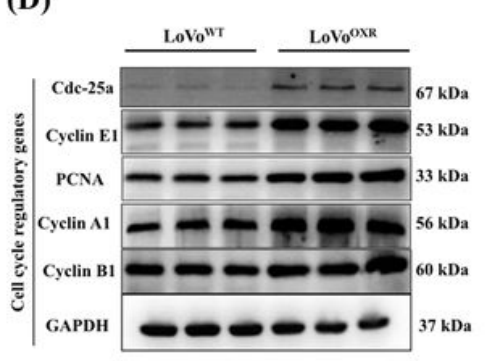

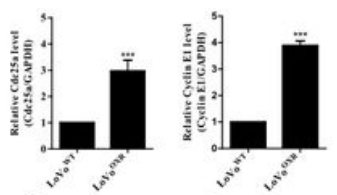

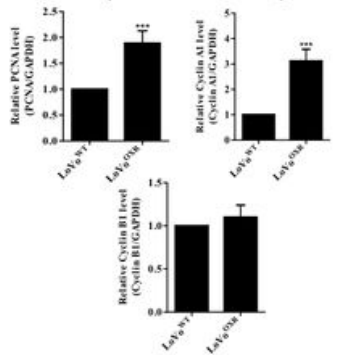

Figure 4

CTDSPL expression is significantly associated with cell cycle alteration in OXR-LoVo cells.

(a) Western blot analysis showing the expression levels of CTDSPL, pRB, RB and E2F1,(b) the cell cycle phase regulatory proteins (Cdc25a, cyclin E, cyclin A, cyclin D1, PCNA, Cdk4, Cdk6 and cyclin B1); (c) cell cycle inhibitory proteins ( $p-p 53, p 15 / 16, p 19)$ in parental and Oxaliplatin-resistant LoVo cells. GAPDH was used as endogenous control and normalized againts in all protein. Quantification of data was carried out by Image J software 1.50 and are presented as the mean \pm SD $(n=3)$. Significance described as $* p<0.05$ vs. Oxaliplatin-resistant LoVo cells. 
Figure 5

(A)
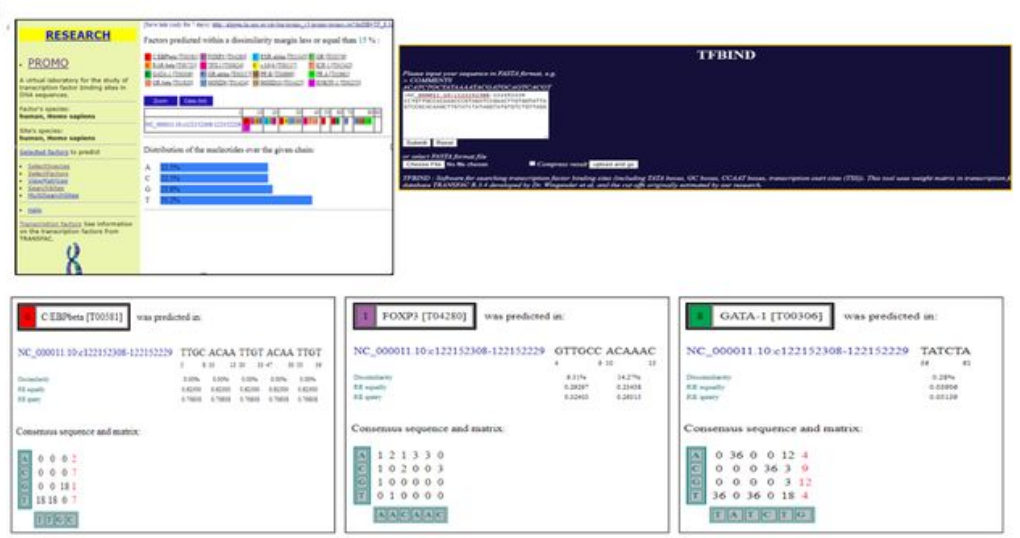

(B)

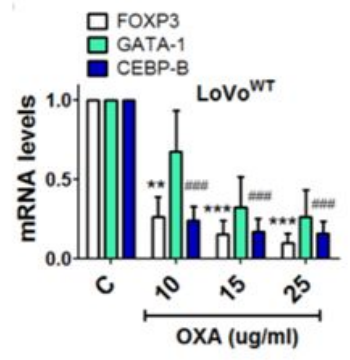

(C)
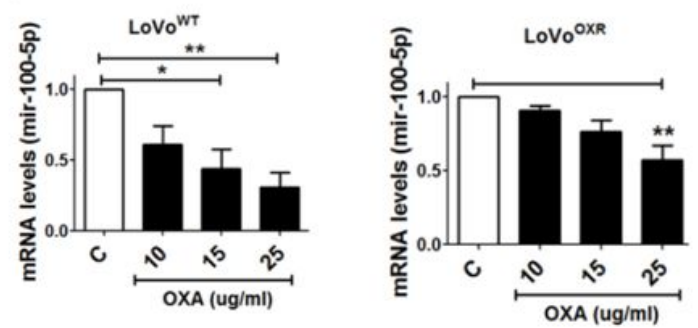

(D)
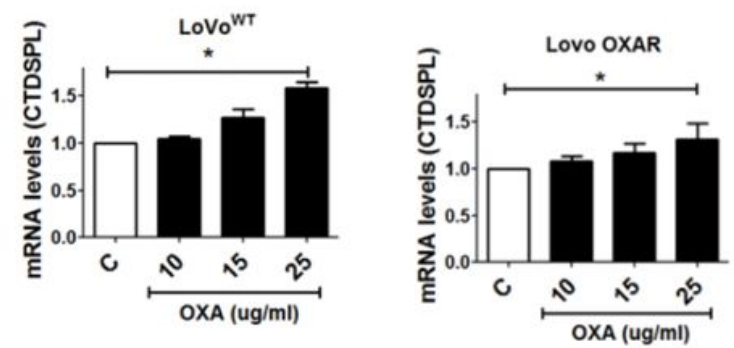

Figure 5

The upstream of miR 100-5p is associated with CTDSPL expression in OXR-LoVo cells.

(a) We used bioinformatic system and dissimilarity index with matrix to screened the upstream of miR100-5p. (b) The mRNA level of OXR-LoVo cells on upstream target (GATA-1, CEBP- $\boldsymbol{\beta}$ and FOXP3) of miR100-5p after treated with Oxaliplatin drug. (c) mRNA level of miR-100-5p after treated with Oxaliplatin in colorectal cancer cell. (d) mRNA CTDSPL expression level is associated with miR-100 5p in colorectal colon cancer cell. 
Figure 6

(A)
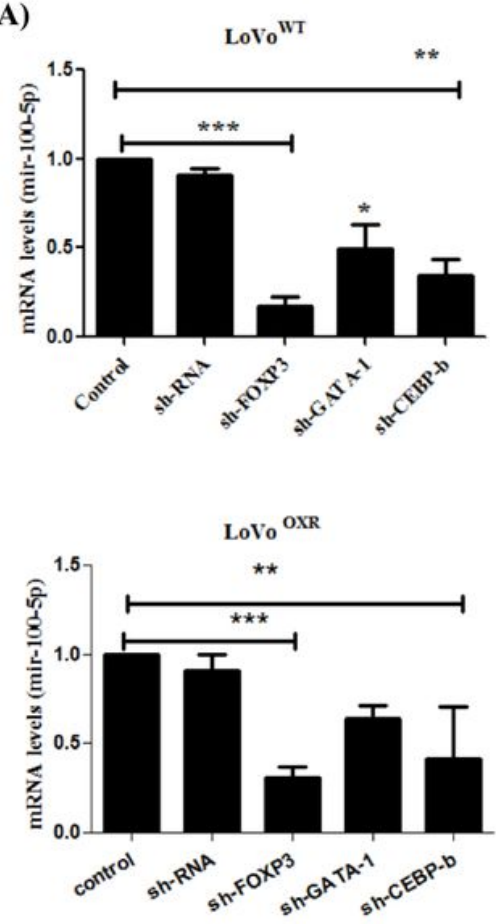

(B)
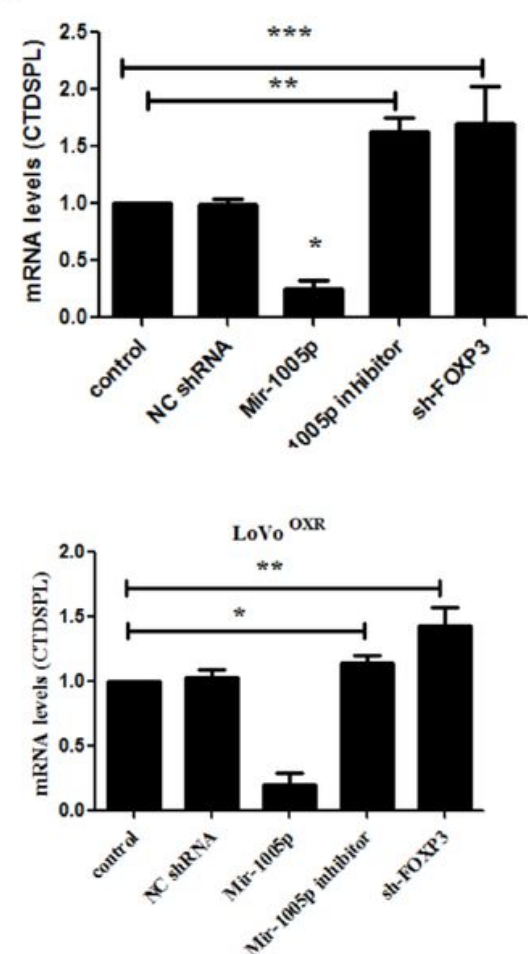

(C)
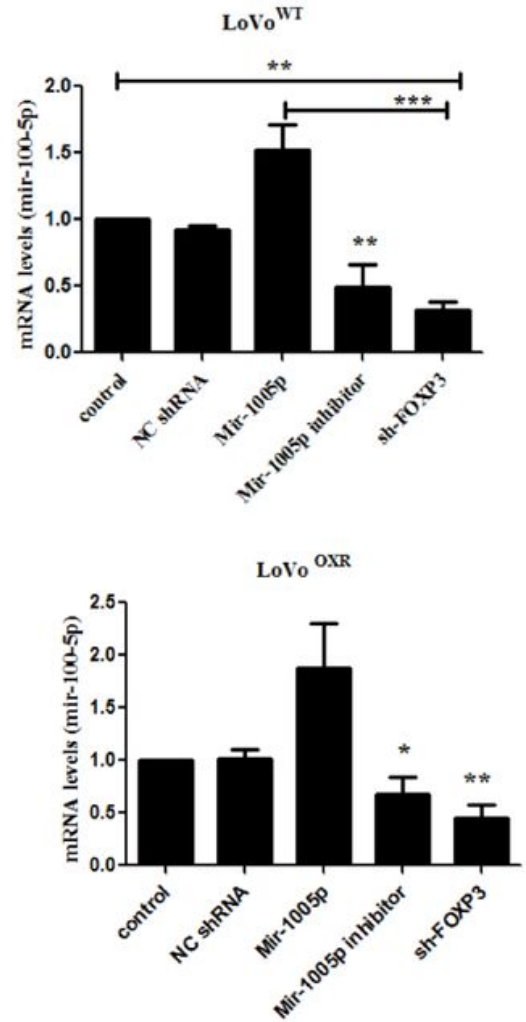

Figure 6

Knockdown the upstream of miR 100-5p in the LoVo parental and LoVo-OXR

(a) We used qPCR method to measure the expression miR-100-5p after treated with three upstream shRNA (sh-FOXP3, sh-GATA-1, sh-CEBPb) in LoVo parental. (b) The expression of miR-100-5p was decreased after treated with sh-FOXP3 in LoVo-OXR and CTDSPL expression is associated with upstream target of miR-100-5p in colorectal cancer cell. (c) FOXP3 was choosen as a candidate upstream target miR-100-5p. sh-FOXP3 knockdown has influenced in the miR-100 -5p expression in the chemoresistant colorectal cancer cell 


\section{Figure 7}

(A)

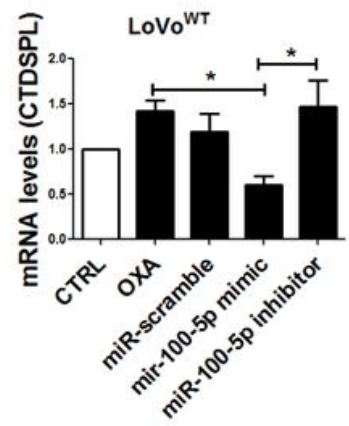

(C)

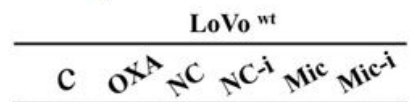

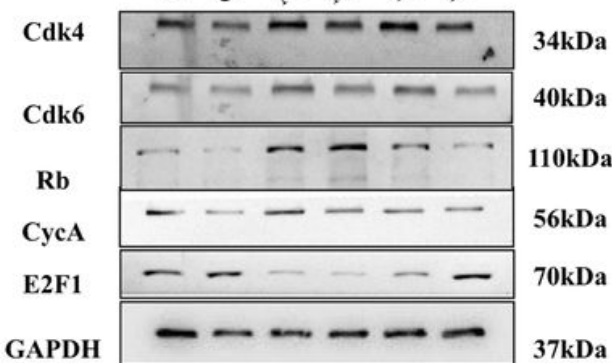

(B)
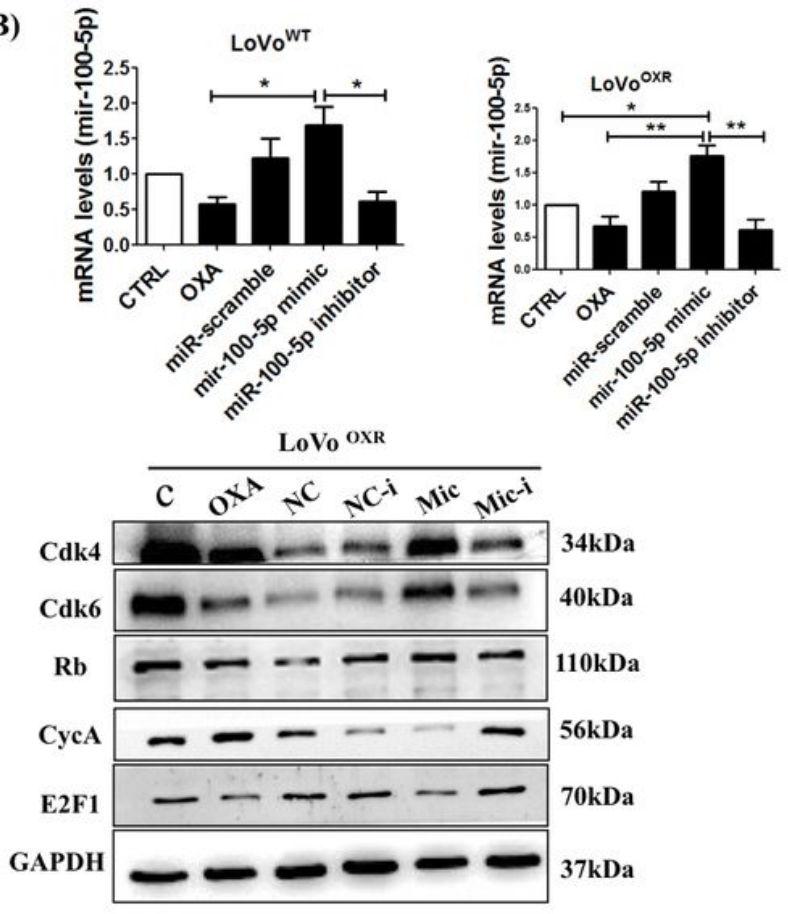

Figure 7

miR-100-5p is targeting CTDSPL as gene target and modulating cell cycle mediated chemoresistance in colorectal cancer cell

(a) Folowing miR-100-5p inhibitor action, the inhibition miR-100-5p was increasing the level of CTDSPL expression and (b) supressed the level of miR-100-5p, (c) also affected in the cell cycle regulator protein level which was decreased in the chemoresistant colorectal cell. 


\section{Figure 8}
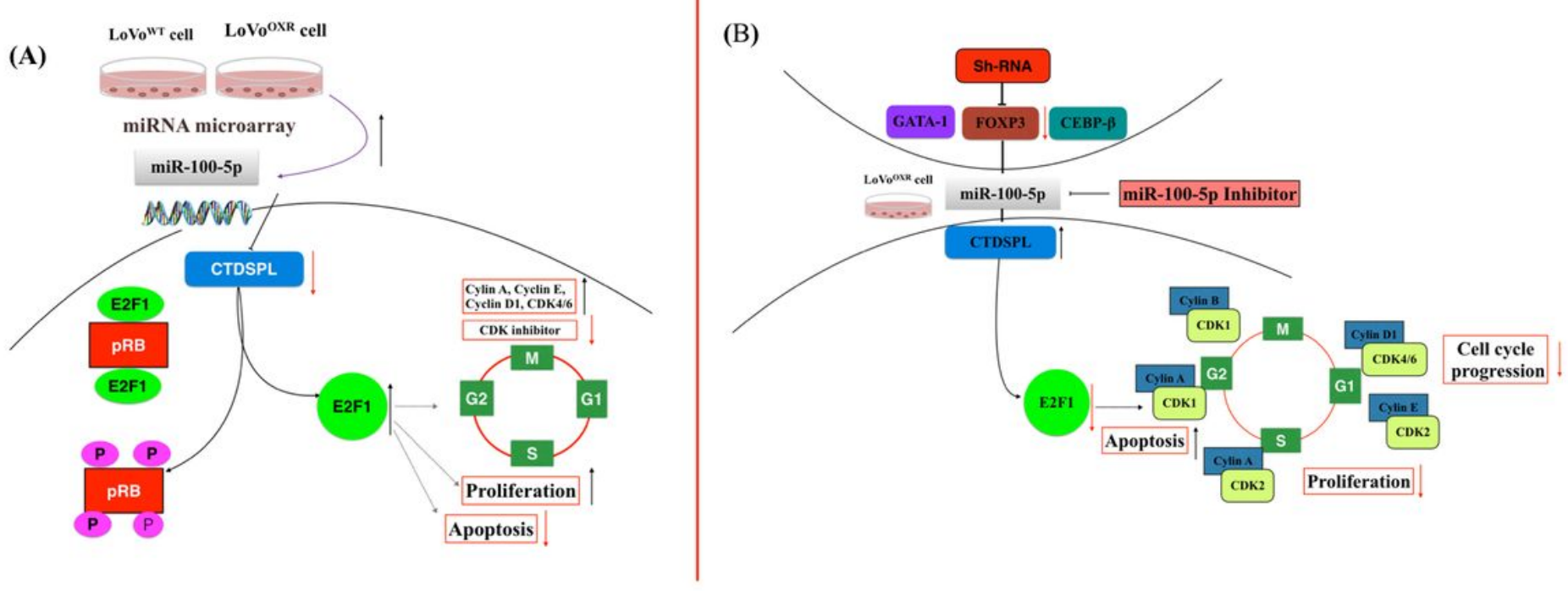

\section{Figure 8}

Proposed mechanism of miR-100-5p enhanced cell cycle mediated chemoresistant through modulating CTDSPL/pRB/E2F1 signaling pathway in CRC. (a) miR-100-5p level is highly expressed in LoVo-OXR meanwhile CTDSPL expression is downregulated. (b) CTDSPL is downstream putative target of miR-100$5 p$ and FOXP3 is upstream target of miR-100-5p in chemoresistant colorectal cancer cell. 